\title{
La profesionalización periodística en Ecuador: ¿la experiencia en las calles o el conocimiento de las aulas?
}

\author{
María Isabel Punín LARREA \\ Universidad Técnica Particular de Loja (UTPL) (Ecuador) \\ mipunin@gmail.com \\ Alison Catherine MARTínez HARO \\ Universidad Técnica Particular de Loja (UTPL) (Ecuador) \\ acmartinez19@hotmail.com
}

Recibido: 31/07/2012

Aceptado: 14/01/2013

\section{Resumen}

El artículo analiza el estado actual del ejercicio periodístico en el Ecuador y las tendencias académicas predominantes dentro de las escuelas y facultades de Comunicación Social en el país, espacios en los cuales se consolida un perfil profesional cuyo rol ha sido puesto en duda. Concluye con un análisis sobre la capacitación, actualización y profesionalización del periodismo ecuatoriano, tomando como referencia una encuesta aplicada al personal con funciones directivas y periodísticas de 2.944 medios de comunicación del Ecuador, dentro de un proyecto de investigación nacional denominado: "Mapa de la comunicación de Ecuador", desarrollado por la Universidad Técnica Particular de Loja.

Palabras clave: Comunicación, Periodismo, Universidad, Ecuador

\section{The Journalistic Professionalization in Ecuador: the Experience on the Streets or the Knowledge of the Classrooms?}

\begin{abstract}
This article discusses the current state of journalism in Ecuador and the predominant academic trends within schools and faculties of Social Communication in the country, spaces in which consolidates a professional profile whose role has been questioned. Concludes with an analysis of the need for training, updating and professionalization of the Ecuadorian journalism, taking as reference a survey applied to personnel with directives and journalistic functions of 2,944 media of Ecuador, within a national research project called: "Map of the communication of Ecuador", developed by the Technical University of Loja.
\end{abstract}

Keywords: Comunication, Journalism, University, Ecuador

\section{Referencia normalizada}

PUNIIN LARREA, María Isabel y MARTÍNEZ HARO, Alison C. (2013): "La profesionalización periodística en Ecuador: ¿la experiencia en las calles o el conocimiento de las aulas?”. Estudios sobre el mensaje periodístico. Vol. 19, Núm. 1, págs.: 505-517. Madrid, Servicio de Publicaciones de la Universidad Complutense.

Sumario: 1. Introducción. 2. La formación de comunicadores sociales dentro del contexto Latinoamericano. 3. La formación del Comunicador Social en Ecuador; 3.1. Comunicadores Sociales con título universitario y sin título universitario. Medios Impresos en Ecuador; 3.2. Comunicadores Sociales con título universitario y sin título universitario. Medios Digitales en Ecuador; 3.3. Comunicadores Sociales con título universitario y sin título universitario. Radio en Ecuador; 3.4. Comunicadores Sociales con título universitario y sin título universitario. Televisión en Ecuador. 4. Comunicación, medios y poder. Un conflicto permanente. 5. La Universidad y los medios pueden mejorar la preparación de los comunicadores y periodistas en el Ecuador. 6. Conclusiones. 7. Referencias bibliográficas. 


\section{Introducción}

El proceso de profesionalización del periodista ecuatoriano se inició de manera formal dentro de las aulas universitarias en la década del 40, en el siglo XX. Uno de los primeros antecedentes del ejercicio periodístico en el país data de 1792, con la circulación del primer periódico ecuatoriano "Primicias de la Cultura de Quito", bajo la inspiración del médico y periodista Eugenio de San Cruz y Espejo. "Espejo, el fundador del primer periódico de nuestra patria, estableció para el periodismo ecuatoriano el espíritu que nos ha sido muy satisfactorio defender: la democracia, la independencia, la cultura" (Pérez, 1970) ${ }^{1}$, por lo cual está considerado como el padre del periodismo ecuatoriano.

La mayor oferta en relación la formación universitaria de comunicadores sociales en Ecuador está en manos de la universidad privada. Se estima que en el país existen aproximadamente 26 escuelas y facultades de Comunicación Social. La titulación pura en Periodismo ha desaparecido, proceso que se inicia en la década del 60, cuando surge la propuesta de CIESPAL relacionado con la formación polivalente.

Una investigación de envergadura nacional realizada por el departamento de Ciencias de la Comunicación Social de la Universidad Técnica Particular de Loja (UTPL), denominada "Mapa de la comunicación de Ecuador" a lo largo de 2012, al personal con funciones directivas y periodísticas de los medios de comunicación en el país, revela que aún existe un vacío en relación a la vinculación laboral de personas con título profesional en los medios de comunicación, especialmente en la radio, pese a que la oferta de formación dentro de las aulas universitarias es una de las más antiguas de Latinoamérica.

Las cifras relacionadas a la cualificación profesional que se detallaran más adelante, permiten reafirmar una tendencia común y de larga tradición en el país, considerar al Periodismo como un oficio de carácter técnico y no como una profesión de corte humanístico, que se gesta en las aulas universitarias.

De oficio o de profesión, el ejercicio del periodismo en el Ecuador pasa por momento complejo, que obliga a los medios como receptores de profesionales y a la academia, como formadores, a realizar una evaluación critica de doble vía. Dos periodistas ecuatorianos, R. Buitrón y F. Astudillo (2005), realizan una radiografía del periodismo en el país, llegando a algunas conclusiones, a partir de su trabajo en los medios:

a.- El sistema educativo es deficiente; el periodista sale de la universidad sin las herramientas intelectuales y metodológicas necesarias, a pesar de su buen olfato periodístico.

b.- El docente dedicado a la formación de comunicadores no tiene la suficiente experiencia en periodismo; lo dejaron hace mucho y no se han actualizado.

1 Ismael Pérez Pazmiño fue el fundador del diario El Universo de Guayaquil, Ecuador. Nació el 30 de junio de 1876 en Machala provincia de El Oro. La cita se tomó de un largo discurso que Pérez Pazmiño pronnció en 1970 y que se ha reproducido en la Revista Bodas de Oro Diario El Universo (1970) 
c.- La deficiencia también es culpa del alumno, pues lee poco; tiene la idea de que el periodismo es "solo fama". No está predispuesto a hacer investigación, ni buscar las historias fuera de la oficina.

\section{La formación de comunicadores sociales dentro del contexto Latinoamericano}

El origen de la formación universitaria de comunicadores sociales en América Latina puede situarse entre 1934 y 1935, cuando se comenzaron a impartir cursos de periodismo a nivel superior en la Universidad de La Plata- Argentina. Las escuelas y facultades en Latinoamérica nacieron emulando el modelo clásico de las escuelas de periodismo que se implementaron en Norteamérica y en Europa. Los centros de formación universitaria se desarrollaron paralelamente al proceso de industrialización. "En América Latina las escuelas de comunicación nacen en Brasil y Argentina en los años treinta, y responden primeramente a la necesidad de formar profesionales para la industria periodística y posteriormente para laborar en los medios en general" (Karam; Canizañez, 2010). El crecimiento de las escuelas y facultades de Comunicación ha sido totalmente sorprendente, aunque no existe un registro oficial sobre este tema. El dato de que dispone la Federación Latinoamericana de Facultades de Comunicación Social -Felafacs 2012- indica que hay 295 escuelas y facultades de Comunicación/ Periodismo."En Latinoamérica, en cuanto al entorno social y profesional del periodismo, hablamos de un profesional pluriempleado, con sueldos escasos y contratos inestables (López, 2005) (Mellado, 2006). Se trata de una situación que en gran medida también está provocada por la abundante oferta de comunicadores sociales que existe en la Región. Dentro del ámbito académico se ha cuestionado la incapacidad de conjugar su proyecto de identidad con las salidas profesionales de sus egresados (Hernández \& Schwarz, 2008). Es un aspecto que aparece como una debilidad frecuente y constante en las ofertas formativas.

Se ha trabajado mucho en el tema de contenidos mínimos que requiere la profesión. Unesco, por ejemplo, presenta toda una oferta para la formación de tercer nivel, denominada: "Plan Modelo de Estudios de periodismo de la UNESCO", cuyo proceso de adaptación curricular ha sido implementado en países como Argentina, Indonesia, España, entre otros ${ }^{2}$. Por otro lado, según Ortega y Humanes (2000), el que podamos referirnos a ideologías profesionales dentro del ámbito de la comunicación implica varios supuestos, que han sido aceptados por la academia como parte de los horizontes formativos profesionales:

1. Que la profesión ha alcanzado un nivel de profesionalización suficiente como para que sus miembros tengan conciencia de compartir una cultura ocupacional.

2. Que la profesión se ha convertido en un campo de acción autónomo con sus propias reglas.

${ }^{2}$ Los datos completos del proceso de adaptación curricular por países y centro de estudio se pueden ser en Mapa de centros y programas de formación para comunicadores. Disponible en: portal.unesco.org/ci/en/files/29317/...es.pdf/mapping_es.pdf. Acceso: 10/06/12 
3. Que compite con otras instancias para ganar influencia social y que para ello necesita legitimarse a través de prácticas que se transforman en modelos de referencia y rutinas de trabajo.

El periodismo, desde sus inicios, ha sido una disciplina ecléctica que se ha nutrido de un sinnúmero de perspectivas teóricas y metodológicas (Mellado, 2006). Esta posición multidisciplinaria sumada a las tendencias del mercado han provocada de la formación de comunicadores tengan dos tendencias bien delimitadas.

La primera, una formación de corte humanística, que considera al periodista un intelectual (Fuentes, 1992) y la segunda, la formación técnica sobre en temáticas relacionadas con la producción audiovisual, el marketing y la comunicación organizacional, que es por cierto las tendencias más frecuentes en Ecuador y en Latinoamérica. En el debate sobre estas dos tendencias, que ha tomado fuerza dentro del mundo académico y no hay acuerdos mínimos. Por un lado está la posibilidad de las desprofesionalización del periodista (Mcleody Rush, 1969; Donsbach y Klett, 1993; Broddason, 1994; Weaver, 1996 y otros) provocado sobre todo por las nuevas tecnologías. Y la segunda propuesta del círculo académico en relación a la profesionalización (Aznar, 1997; Humanes, 1998).

\section{La formación del Comunicador Social en Ecuador}

El proceso de formación universitaria de comunicadores sociales en nuestro país nace en la década del 40, con la creación de la primera Facultad de Comunicación Social, por parte de la Universidad Central del Ecuador (1943), en la ciudad de Quito, y luego con la formación de la Escuela de Información de la Universidad Estatal de Guayaquil (1945), en la ciudad de Guayaquil. Existe un dato muy importante, como lo recuerda el académico José Villamarín Carrascal. Es el hecho de que en 1975, el General Rodríguez Lara emitió la Ley del Ejercicio Profesional del Periodista, documento que cita: cualquier ciudadano que avale 5 años de experiencia podía acceder a un título profesional, pese a la existencia de centro de formación universitaria, antes señalados.

La citada Ley de Ejercicio Profesional, promulgada en el Registro Oficial No. 900 de 1975, expresa de manera textual en el Art. 1, lo siguiente: "Es periodista profesional literal c.) "Quien obtuviera un certificado de profesionalización otorgado por el Ministerio de Educación Pública, en razón de hacer ejercicio la profesión, con anterioridad a la fecha de vigencia de esta Ley, y de acuerdo con sus disposiciones..." (Salvador, 2006).

La exigencia de un título universitario para el ejercicio del periodismo o cualquiera de las ramas de la comunicación en Ecuador era un "tema muerto" hasta la llegada al poder del presidente Correa. Especialmente en su segundo mandato, se evidencia un discurso provocador que hiere el trabajo y rol de la prensa en el país, en particular, lo que ha reabierto el debate sobre la titulación y la formación.

Esta posición se suma a una crisis de la educación ecuatoriana, que ha obligado a la sociedad a exigir una evaluación sobre la calidad de la educación superior, en este caso de comunicación. 
Una medida puntual del gobierno de Rafael Correa ha sido la implementación de un proceso de evaluación y acreditación de la Universidad Ecuatoriana, categorizando a las instituciones de educación superior en A, B, C, D y E.

En la categoría A, es decir con la máxima en su evaluación, están 11 universidades; de estas 11 universidades, 7 de ellas ofrecen la carrera de comunicación social y/o sus otras vertientes del conocimiento y aplicación. Las universidades en el grupo A son:

1. Pontificia Universidad Católica de Quito

2. Universidad Central del Ecuador

3. Universidad de Cuenca

4. Universidad del Azuay

5. Universidad San Francisco de Quito

6. Universidad Técnica de Ambato

7. Universidad Técnica Particular de Loja

En la categoría B se ubicarían también 7 universidades que cuentan con escuelas y facultades de Comunicación:

1. Universidad de Guayaquil

2. Universidad Nacional de Loja

3. Universidad Estatal de Bolívar

4. Universidad Nacional de Chimborazo

5. Universidad Politécnica Salesiana

6. Universidad Técnica Del Norte

7. Universidad de Las Américas

El resto de universidades, que son las que no se encuentran ubicadas en capitales de provincia, se sitúan en la categoría $\mathrm{C}$ y $\mathrm{D}$; algunas han ido clausuradas debido al incumplimiento de los estándares de calidad impuestos por el Gobierno, según los resultados de la evaluación citada.

La propuesta formativa de comunicadores sociales del Ecuador fue siempre la "niña mimada" de la región. No podemos olvidar que el Centro Internacional de Estudios Superiores de Comunicación para América Latina (CIESPAL) jugó un rol protagónico dentro del mundo académico a lo largo de la década de los 70's.

CIESPAL lideró el proyecto de formación polivalente, que permitió en primera instancia ampliar el perfil profesional, estimulando a su vez el crecimiento de las facultades y escuelas de comunicación en toda Latinoamérica, aportando con mano de obra cualificada.

Al hablar del contexto académico latinoamericano no se puede dejar de mencionar a la primera escuela de periodismo en América Latina, Universidad Nacional de la Plata (Argentina). A partir de ello podemos citar tres momentos que han marcado el proceso formativo de la comunicación, cuyos escenarios han sido más los medios de comunicación que las aulas.

El proceso de formación académica y empírica de Comunicadores en Latinoamérica tiene su historia, que ha sido revisada de manera particular por (Marques de Melo, 
1984; Fuentes, 1997; Mata, 2010; Mellado, 2010), y que a manera de resumen podemos citar así:

a.- 1930-1960: Temporada en la cual los académicos se centran en análisis de los medios, la producción informativa y el comportamiento de las audiencias.

b.- 1945-1969: La creación y consolidación de CIESPAL “[...] surge en la coyuntura latinoamericana como una iniciativa de la UNESCO para diseminar modelos destinados a la preparación de profesionales para los medios de comunicación colectiva, que atienden a las nuevas exigencias socioculturales" (Marques de Melo, 1984), y que aportan a la consolidación una etapa considerada como científico/técnica (Esteinou, 2002).

Dentro de los aportes tangibles de este organismo están los siguientes: Las escuelas deberían tener un nivel académico; una duración mínima de 4 años e incluir enfoques humanísticos y técnico profesionales.(Andión, 1991)

c.- La década de los setenta es cuando los centros de formación universitaria trabajan en la división y subdivisiones de especializaciones. Esta época constituye la temporada de la proyección y afianzamiento de los centros dedicados a la investigación.

Hoy el proceso de formación de comunicadores sociales vive un cuarto momento provocado básicamente por la metamorfosis del periodismo. Recordemos que Internet y la tecnología digital actúan como una centrifugadora imparable y paradójica (Fogel; Patiño, 2007) cuya incidencia en el periodismo es notoria. Y hay una conclusión certera "el periodismo es periodismo al margen de los soportes" (López, 2011)

La consolidación del periodismo ciudadano (Gillmor, 2006) es otro punto a tener en cuenta. Estas tendencias importantes nos plantean otras dudas y cierta confusión, porque actualmente“... es cada vez más difícil definir qué se espera del trabajo de un periodista" (Rosen J, 1996).

Este fenómeno ha revitalizado el rol y las responsabilidades del periodismo frente a una imparable producción de contenidos por parte de los ciudadanos. Los medios tradicionales se enfrentan, con cada revolución tecnológica, a la apremiante tarea de reinventarse para sobrevivir. Hoy los medios somos todos, pero periodistas solamente algunos (Orihuela, 2006).

Dentro del ámbito latinoamericano y también recurrente en el Ecuador se cuestiona el rol que las escuelas de periodismo y comunicación adquieren en torno a las dinámicas que dan forma a la profesión, producto de su falta de definición académica y de su escasa vinculación con la sociedad (Barbero, 1989). De esta tendencia podría derivarse en un problema mayor, porque hoy "las escuelas de periodismo y las facultades de comunicación hoy se enfrentan a la dificultad de unir de manera armoniosa el contenido de las técnicas periodísticas, con las bases teóricas y el estudio de la comunicación social" (Mellado, 2010) y las exigencias que plantean la presencia y acceso a las nuevas tecnologías.

Pese a todas estas posturas y al debate existente, es importante concluir que la enseñanza del periodismo y/o de la comunicación "debe basarse en algo más que la ignorancia, los prejuicios y la estrechez de miras sobre la cultura moderna" (Buchinghan D, 2009), lo cual es y será una reto para la universidad en general. La investigación referida analiza la presencia del recurso humano dentro de 101 medios impresos, 41 medios televisivos, 591 medios radiales y en 15 medios digitales del país. En gene- 
ral, en el país, de los 101 medios impresos tomados en cuenta en el análisis, se constata que existe un mayor número de titulados, con 1321 profesionales, con titulo de tercer nivel, por sobre los no titulados con 1261 personas.

En relación a los medios impresos, se observa que la presencia de periodistas profesionales vinculados a este sector es ligeramente superior a los no titulados. En los medios impresos, las tres provincias con mayor porcentaje de personas con título de tercer nivel son: Cotopaxi, con el $100 \%$ de sus empleados titulados, le sigue Azuay con el 72, $59 \%$, y Sucumbíos con el 71, $43 \%$. Estos datos son relevantes porque en las poblaciones antes citadas no se alojan de manera mayoritaria las ofertas de estudio.

Las provincias con menor número de profesionales son: Zamora Chinchipe, que ocupa el $100 \%$ de empleados no titulados, pese a su cercanía con la ciudad de Loja, donde se ubican dos universidades que cuentan con la oferta de estudios en comunicación.

En la segundo ubicación, de acuerdo al número de personas sin título, está Santo Domingo, con el $75 \%$, y Carchi e Imbabura, con el $71.43 \%$, ambas provincias donde no se ubican ninguna oferta en categoría A.

\subsection{Comunicadores Sociales con título universitario y sin título universitario. Medios Impresos en Ecuador}

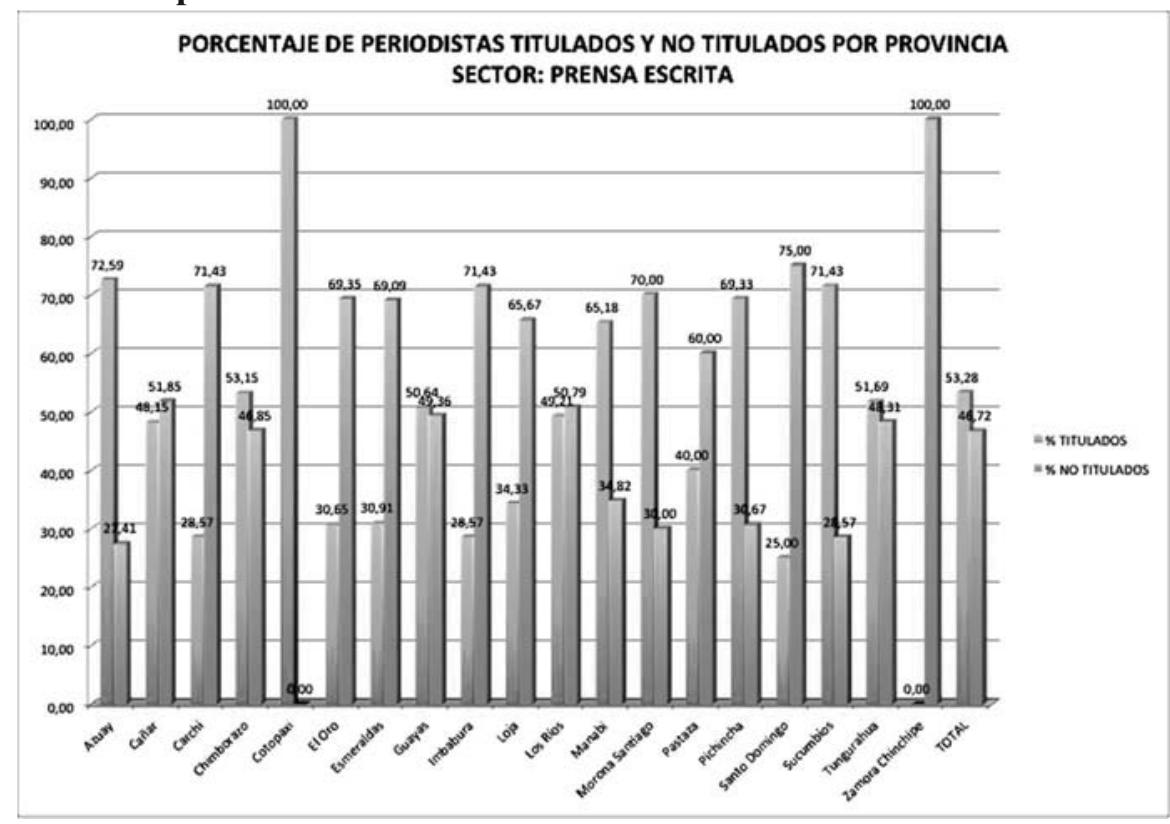

Elaboración propia

\subsection{Comunicadores Sociales con título universitario y sin título universitario. Medios Digitales en Ecuador}

Para este sector se tomó como referencia una muestra total de 15 medios digitales, considerando únicamente aquellos medios que tienen más de 3 años de permanencia 
en el mercado. Bajo está delimitación, los medios de referencia dentro del espacio digital se ubican en 5 provincias del Ecuador. Este sector está marcado por la exigencia de un título universitario; dentro de las 5 provincias, un total de 87 titulados sobre 34 personas sin título. El mayor número de titulados está en la provincia de Azuay, con el 83,33\%, donde ocupa un lugar destacado el siguiente sitio: www.elmorlaco.com; en esta región además existen dos ofertas académicamente, un privada y otra pública, tradicionalmente fuertes.

En un segundo lugar está la provincia de Pichincha, con el $73 \%$; en contraste la provincia con menos número de profesionales con título está en Guayas, con el 100 $\%$,y en Manabí, con el 42,86 \%. En el cuadro a continuación asoman únicamente los medios digitales del país; no se han considerado los medios impresos, por ejemplo que tienen de manera permanente una página web, por formar parte del grupo del grupo relacionado netamente con medios impresos.

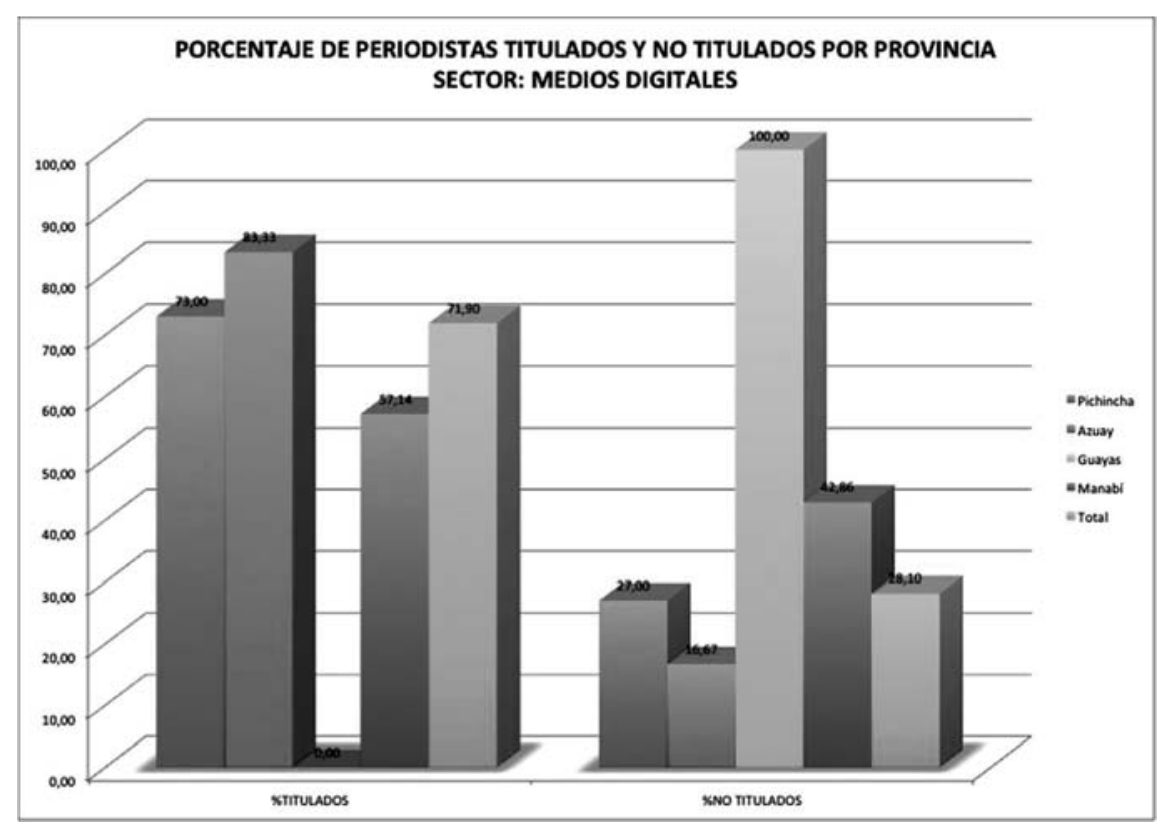

Elaboración propia

En cuanto al medio radio, es la muestra más extensa de toda la investigación. Se tomó como referencia a un total de 591 radios de todo el país, de 23 provincias del Ecuador. Se constata que existe un menor número de titulados, con 1881 profesionales, con titulo de tercer nivel, inferior a los no titulados, con 2375 personas.

La radio es el sector que menos cantidad de profesionales titulados, según los datos que han sido reportados por el personal vinculado con dicho sector. Es, además, uno de los medios en los cuales los mecanismos de vinculación laboral son informales y en muchos de los casos temporales. 


\subsection{Comunicadores Sociales con título universitario y sin título universitario. Radio en Ecuador}

Con respecto a las radios del país, las tres provincias con mayor número de profesionales son: Tungurahua con el 57,31\%; Pichincha tiene el 54,91\%, y Cotopaxi con el $50,52 \%$. Las tres provincias con mayor porcentaje de no titulados son: Carchi, con el $82,35 \%$; Santo Domingo, con el $80 \%$, y Sucumbíos, con el 79,07\%.

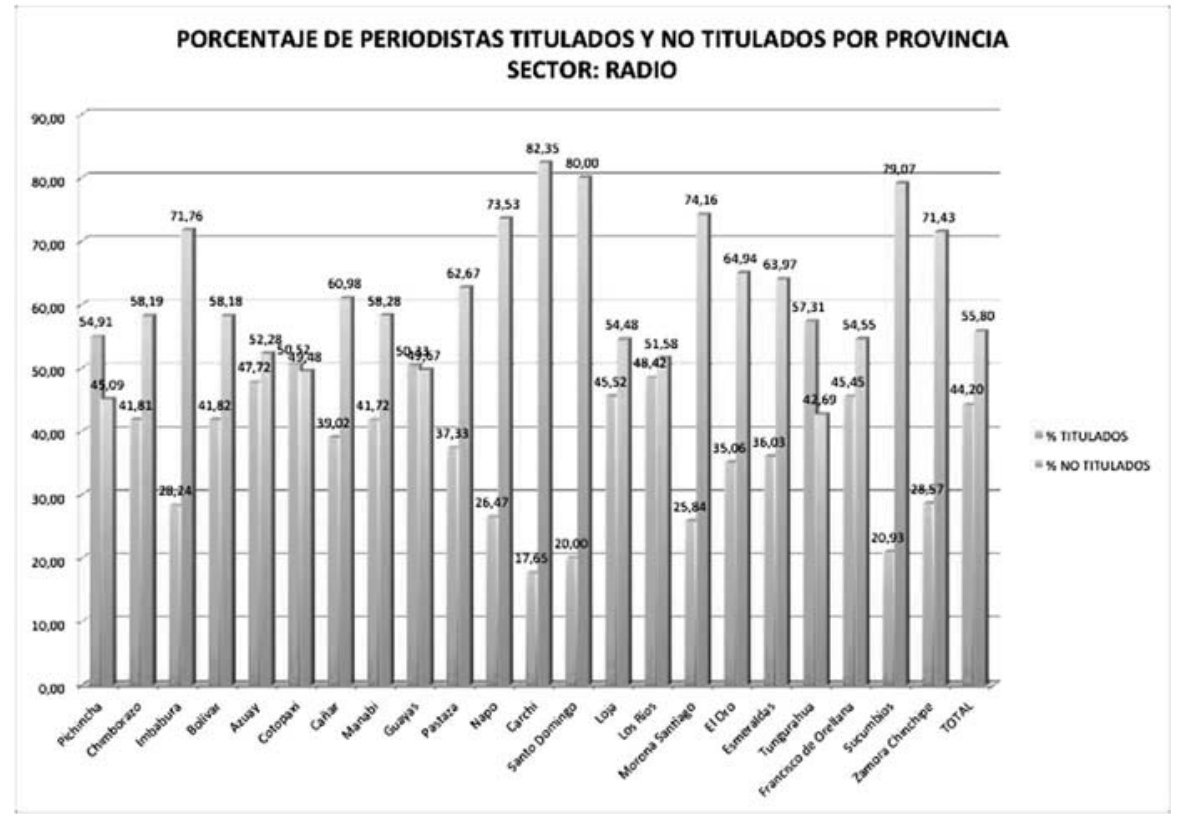

Elaboración propia

\subsection{Comunicadores Sociales con título universitario y sin título universitario. Te- levisión en Ecuador}

En cuanto al medio audiovisual, la televisión se tomó como referencia a 16 provincias del Ecuador, resultando inferior el número de titulados con 566 profesionales, con titulo de tercer nivel, en comparación con las 677 personas no tituladas de estos medios.

De la televisión ecuatoriana, las tres provincias con mayor número de profesionales se destacan: Loja, con el 76,04 \% que tiene ofertas académicas en A y B; seguida de Pichincha, con el 66, $95 \%$, y Tungurahua, con el 62,79 \%. Con relación a los no titulados, las tres provincias con mayor porcentaje son: Los Ríos, con el 83,33 \%; Cotopaxi con el $82,14 \%$, y Guayas, con el 72,78\% 


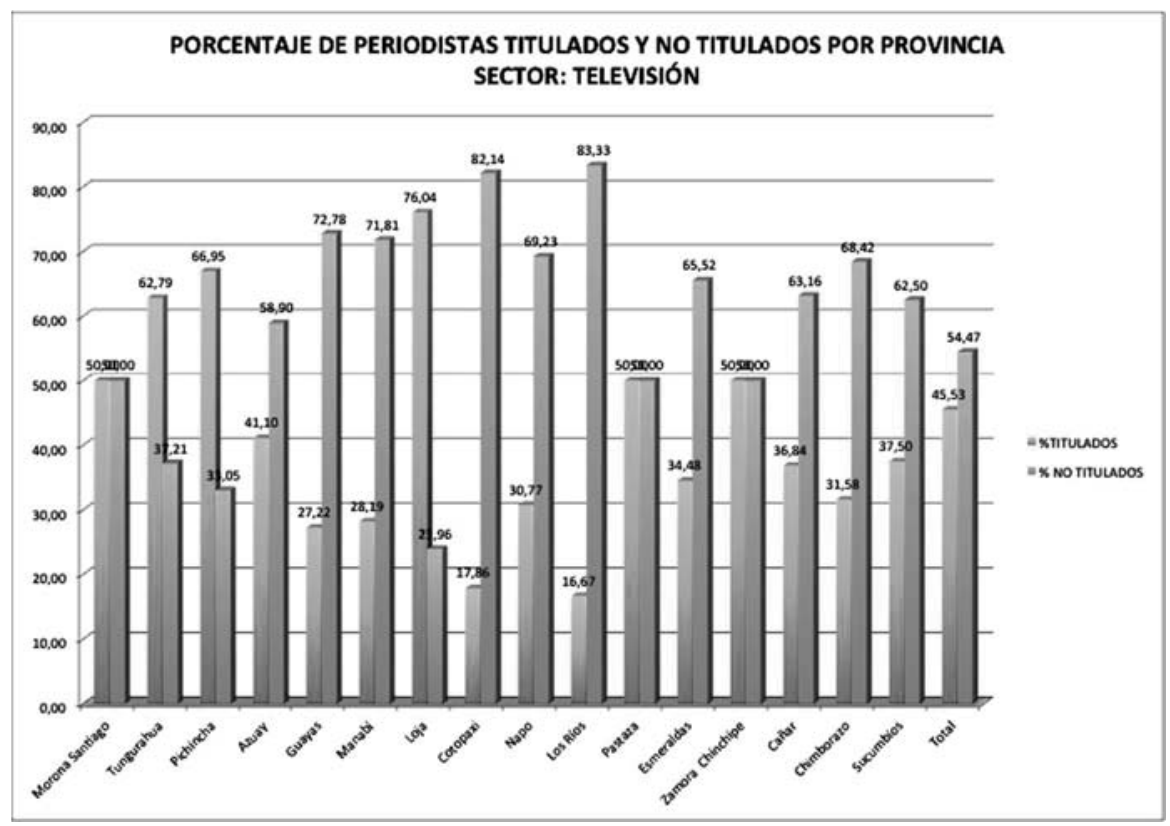

Elaboración propia

\section{Comunicación, medios y poder. Un conflicto permanente}

Para tener una dimensión real de este punto, es importante dar un vistazo a la estructura mediática del país, sobre todo a los grandes grupos empresariales. Es por ello que debemos indicar que en el Ecuador existe algo más de 2.900 medios de comunicación, esto según un mapeo que realizó el Departamento de Ciencias de la Comunicación de la UTPL y que hemos hecho referencia continua en este artículo.

La gran estructura mediática del país, es decir los medios de influencia nacional, está compuesta por dos vertientes: los medios privados propiedad de los principales grupos empresariales del país, cuya estructura es monopólica, liderando por ocho familias, y en segundo lugar medios públicos de reciente creación, bajo la tutela del gobierno nacional, que lucha por captar la atención de la audiencia. Con relación a las familias que lideran los medios privados están:

- Canal de televisión Teleamazonas, Revistas Dinediciones, Agencia de publicidad Delta, Revistas: Mundo Diners, Gestión, Fucsia, Soho pertenecen al Grupo Egas Grijalva. ETV Telerama, Radio, Diario El Mercurio propiedad del Grupo Eljury. Diario El Comercio, Agencia Servipublicidad, Radio Quito, Radio Platinum, Ecuadoradio, Vespertino Ultimas Noticias, Revista Líderes y Familia al Grupo Mantilla. Caravana TV, Radio Caravana Grupo Caravana.

- Diario La Hora, Radio Planeta, Revista Judicial, Satnet (Servicios Agregados y Telecomunicaciones) propiedad del Grupo Vivanco. Diarios Extra y Expreso pertenece al Grupo Martínez. Periódicos El Universo y Súper y Radio City son 
propiedad del Grupo Pérez. Y Canal Ecuavisa, Revistas Vistazo, Generación 21, Hogar, Estadio son propiedad del Grupo Alvarado Roca ${ }^{3}$.

Respecto a los medios públicos existen dos divisiones, los medios incautados y los medios creados bajo la presidencia de:

El Telégrafo, Gama TV, Canal UNO, TC Televisión, Cable Visión, Radio Super K 800, Radio Carrusel, Radio La Prensa, Multicom, Cable Noticias CN3, Cable deportes CD7, Radio El Telégrafo, Revista La Onda, Revista El Agro, Revista El Valle, Revista Samborondón, fueron incautados del Grupo Isaías.

En cambio Ecuador Tv (ECTV), Radio Pública, Agencia de Noticias Andes, Rotativo Interino El Ciudadano, Periódico Popular PP ELVERDADERO fueron creados durante el gobierno de Rafael Correa.

Los medios comerciales son considerados por el régimen como "corruptos y mentirosos". Existe además el pedido expreso del Presidente Correa a sus ministros y funcionarios de Estado, de no brindar declaraciones a medios tildados como corruptos ${ }^{4}$. "El periodista no es simplemente un asalariado; es, por regla general, un hombre de opiniones y convicciones y las emplea en su trabajo" (Jones, 1980). Este espacio de libertad podría minarse en el país, debido principalmente a la estructura y propiedad de los medios y a un nuevo autor el Gobierno.

Un informe de Fundamedios (2012), por intermedio de la Red de Monitoreo de Amenazas a la Libertad de prensa, revela un alto incide del hostilidad para el ejercicio del periodismo en el país. El informe del primer trimestre del 2012 registra 53 agresiones contra medios de comunicación, periodista y ciudadanos.

\section{La Universidad y los medios pueden mejorar la preparación de los comunica- dores y periodistas en el Ecuador}

El proceso de preparación, capacitación y actualización de comunicadores en el país está rodeado de intentos aislados de gremios, fundaciones y, en alguna medida, las universidades. Cada uno cuenta con su propuesta, con un plan de gestión, pero separados. Los intentos más visibles fuera de la academia están de mano de Fundamedios, organización no gubernamental activa desde el 200 que intenta promover y proteger los derechos y libertades en torno al periodismo, entre otras. La Fundación ha soportado las más duras críticas por parte del régimen, sobre todo por el origen de los fondos económicos que la financian. Es importante reconocer que 10 años atrás los medios de comunicación, como diario El Universo y El Comercio, habían implementado fundaciones paralelas a los medios con el objetivo de capacitar a su personal; sin embargo estas iniciativas se han ido borrando a lo largo de la historia.

3 Datos: Informe de la Comisión para la Auditoría de Frecuencias de Radio y Televisión. Disponible en: http://www.telegrafo.com.ec/index.php?option $=$ com_zoo\&task $=$ item\&item_id $=38160 \&$ Itemid $=2$. Acceso: $02 / 05 / 12$.

4 La nota se difundió el pasado 9 de junio del 2012 y puede visualizarse en la siguiente dirección: http://knightcenter.utexas.edu/es/node/10432 
En Ecuador, al igual que otros países del continente, existen acuerdos para defensa de la libertad de expresión la unión de los periodistas, de todos ellos, es la única forma posible de avanzar en la defensa de los intereses, los bienes y los valores de su profesión (Aznar, 1997). Anhelo que estaría respaldado, por la declaración de derechos humanos art. 19 y por los art. 16, 18 y 66 de la Constitución de la República del Ecuador, entre otros.

La anunciada Ley de Comunicación, de aprobarse, velaría, por ejemplo, por el ejercicio profesional del periodismo; sin embargo, en ello, tampoco hay un consenso de la clase política de nuestro país. La relación gremios profesionales y la academia se reduce a actividades muy puntuales.

\section{Conclusiones}

El ejercicio del periodismo en Ecuador pasa por un momento difícil porque no ha podido escapar de la crisis mundial que afecta al prestigio y a la independencia del periodista y del periodismo, en particular.

Los periodistas ecuatorianos ejercen su profesión en un escenario complejo, marcado por una confrontación permanente entre el Presidente Correa y la empresa mediática privada. Las acusaciones de ambos grupos han sido fuertes.

La propuesta formativa universitaria relacionada con las ciencias de la comunicación es de larga tradición en el país y, en general, los centros universitarios han optado por una formación de carácter inter y trans disciplinaria.

El proyecto de investigación "Mapa de la Comunicación de Ecuador" nos revela que el sector en el cual existe mayor necesidad de capacitación y titulación de tercer nivel está en la radio, que es además uno de los sectores más amplios en el país.

La mayor cantidad de periodistas titulados está en los medios digitales, en los cuales la demanda de competencias técnicas digitales es superior.

En cuanto al sector prensa escrita y televisión, se evidencia algunas provincias en los cuales la capacitación y la titulación por parte de la universidades han tenido un efecto positivo, como por ejemplo la provincia de Loja.

Pese a la larga existencia de ofertas de educación superior, aún existen sectores que prefieren ejercer el periodismo como un oficio en Ecuador.

\section{Referencias bibliográficas}

ANDION, Mauricio (1991). "La formación de profesionales en comunicación”. Diálogos de la comunicación. Vol. 31, pp. 55-63.

AGUINAGA, Enrique de (1980): Periodismo profesión. Madrid, Fragua.

ASTUDILLO, Fernando y BUITRON, Rubén (2005): Periodismo por dentro. Ciespal, Intiyan Ediciones.

AZNAR, Hugo (1997): "El debate sobre la profesionalización del Periodismo: de la titulación a la organización". ZER, vol.2, n 3, pp. 129-152

BUCHINGHAM, David (2009): "In defense of media studies". Disponible en: http://www.guardian.co.uk/commentisfree/2009/. [Acceso: 10/06/12]. 
ESTEINOU MADRID, Javier (2002): "CIESPAL y la formación de imaginarios de la comunicación en América Latina". Razón y Palabra, No. 25. Disponible en: http://www.razonypalabra.org.mx/anteriores/n25/jesteino.html. [Acceso: 10/06/12]

FRANCO, Guillermo (2007): "Repensar las Facultades de Periodismo". Disponible en: http://www.ecuaderno.com/2007/02/13/repensar-las-facultades-de-periodis [Acceso: 13/06/12]

FOGEL, Jean-François y PATIÑO, Bruno (2007): La prensa sin Gutenberg. El periodismo en la era digital. Madrid, Punto de lectura.

FUNDAMEDIOS ( 2012): "Informe especial. Monitoreo de amenaza a la liberta de prensa. Alto clima de hostilidad en contra de periodistas y medios en el primer trimestre del año". Disponible en: http://www.fundamedios.org/portada/libertades/alertas.html. [Acceso: 15/07/12]

FUENTES NAVARRO, Raúl (1988): "Escuelas de Comunicación y brechas tecnológicas en México". Diálogos de la Comunicación. Número 19. Enero, p. 37.

GILLMOR, Dan (2006): "We the media: Grassroots journalism by people, for the people". Sebastopol: O’Reilly Media. Inc. Disponible en: http://www.oreilly.com/cata$\log /$ wemedia/book/index.csp

JONES, J. Clement (1980): "Mass Media Codes of Ethics and councils. Acomparative internacional study on profesional standards". Paris, Unesco Press.

KARAMUS, Tanius y otros (2010): "Veinte formas de nombrar a los medios masivos. Introducción a enfoques, modelos y teorías de la comunicación". Universidad de Los Andes.

LOPEZ HIDALGO, Antonio (2005): El periodista en su soledad. México, Alfaomega.

LOPEZ, Xosé (2011): Metamorfosis del periodismo. Ecuador, UTPL.

MARQUES DE MELO, José (2009): Pensamiento comunicacional latinoamericano. Entre el saber y el poder. Cap. 18: "Años setenta: la resistencia crítica”, pp. 285296. Sevilla, Comunicación Social, Ediciones y Publicaciones.

MELLADO, Claudia (2006): Balance 2006. Perspectivas profesionales y del mercado periodístico de la Octava Región. Concepción. Universidad de Concepción, Chile, Publicaciones Dirección de Docencia.

ORIHUELA, José Luis (2006): "Iniciativa de la comunicación. Entrevista con José Luis Orihuela". Disponible en: http://www.comminit.com/la/node/67584. [Acceso: $11 / 06 / 12]$.

ROSEN, Jay (1996): Getting the connections right public Journalism and the troubles in the press- Twentieth Century Fund Press/ The Eletronic Policy Network.

SALVADOR GARCÍA, Milton (2006): Periodistas sin título. Quito, Ecuador, Editorial Soboc Grafic. 